\title{
Endoscopic Total Parathyroidectomy and Partial Parathyroid Tissue Autotransplantation for Patients with Secondary Hyperparathyroidism: A New Surgical Approach
}

\author{
Yueming Sun · Huihua Cai · Jianfeng Bai • \\ Hanlin Zhao • Yi Miao
}

Published online: 17 June 2009

(c) The Author(s) 2009. This article is published with open access at Springerlink.com

\begin{abstract}
Background Secondary hyperparathyroidism (SHPT) (i.e., renal hyperparathyroidism) is one of the most serious complications in long-term hemodialysis patients. The purpose of this retrospective study was to explore the feasibility of a new surgical approach-endoscopic total parathyroidectomy with autotransplantation (ETP+AT) — and evaluate its practical application for patients with SHPT.

Methods The study included 34 SHPT patients who underwent ETP+AT from among 67 cases at the Department of Minimally Invasive Surgery, the First Affiliated Hospital of Nanjing Medical University over a 3-year period. The other 33 patients underwent traditional total parathyroidectomy with autotransplantation $(\mathrm{TP}+\mathrm{AT})$. Two criteria were used as indications to perform ETP+AT in SHPT patients. The first was a high serum parathyroid hormone level (PTH $>800 \mathrm{pg} / \mathrm{ml}$ ) associated with hypercalcemia and/or hyperphosphatemia that which were refractory to medical treatment. The second criterion was the presence of clinical symptoms including pruritus, bone and joint pain, muscle weakness, progression of soft tissue calcification, and spontaneous fractures. Ultrasonography, ${ }^{99 \mathrm{~m}} \mathrm{Tc}$ sestamibi scans, and computed tomography were used to evaluate the thyroid and parathyroid glands.

Results There was no surgery-related mortality among any of the patients with ETP+AT. One patient underwent conventional neck exploration because of bleeding and
\end{abstract}

Y. Sun $(\varangle) \cdot$ H. Cai · J. Bai · H. Zhao · Y. Miao Department of Minimally Invasive Surgery, First Affiliated Hospital of Nanjing Medical University, 300 Guangzhou Road, Nanjing, Jiangsu 210029, China

e-mail: jssym@vip.sina.com

H. Cai

e-mail: chh1168@163.com injury of a unilateral recurrent laryngeal nerve after the operation. Preoperative symptoms were alleviated, and the serum PTH and alkaline phosphatase levels, hyperphosphatemia, and hypercalcemia were improved or normalized in most patients. Recurrence was observed in one patient with a sixth parathyroid gland behind his thyroid, and the patient required a second operation. Hypoparathyroidism was not found after the operation. The clinical data were compared between ETP+AT and TP+AT.

Conclusions ETP + AT is a safe option for the treatment of SHPT with low morbidity and mortality, shorter hospital stay and low recurrence rate. It is important to avoid intraoperative bleeding, identify all parathyroid glands during the surgery, and choose adequate parathyroid tissues for autografting.

\section{Introduction}

Secondary hyperparathyroidism (SHPT) is a common complication of end-stage renal failure. Despite improved medical management, $0.7 \%$ to $1.4 \%$ of dialysis-dependent patients require parathyroidectomy annually [1], The association between parathyroid hyperplasia and chronic renal failure (CRF) has been known since the early 1930s [2]. SHPT is an adaptive response to CRF with disruptions in the homeostatic control of serum phosphorus, calcium, and vitamin D. High levels of parathyroid hormone (PTH), phosphate, and the calcium-phosphate product in the circulation system is associated with uremic bone disease, vascular calcification, and death [3, 4]. It has also been suggested that hyperplasia of parathyroid glands occurs during the early stages of renal disease even before dialysis is performed [5]. 
Several medical therapeutic options for SHPT are currently available, with the overall purpose of reducing the serum PTH and phosphate levels, elevating the serum calcium level, and improving skeletal turnover [5-7]. However, several studies have demonstrated that most patients with extremely high serum PTH levels fail to respond to vitamin $\mathrm{D}$ treatment, and surgical operations are required [7, 8]. Parathyroidectomy can significantly decrease the PTH and phosphate levels but is typically performed for patients with refractory SHPT. Parathyroidectomy is required for approximately $10 \%$ to $30 \%$ of the patients with more than 10 years of hemodialysis [9].

In the present study, we retrospectively analyzed 34 hemodialysis patients who were subjected to endoscopic total parathyroidectomy with autotransplantation (ETP+ AT) in our department over a 3-year period. Specifically, here we identify the indications to perform the surgery for SHPT patients, describe the procedure of the surgery, and evaluate the prognosis by biochemical and imaging analysis.

\section{Materials and methods}

Between June 2004 and June 2007, we performed $\mathrm{ETP}+\mathrm{AT}$ in 34 patients with advanced renal hyperparathyroidism (SHPT) and total parathyroidectomy with autotransplantation (TP+AT) in 33 patients with SHPT, who comprised the control group. Hemodialysis was continued for all the patients during this period.

The study group included 15 women and 19 men with a mean \pm SD age of $48.7 \pm 3.5$ years. The average period of hemodialysis before the surgery was 98 months. CRF was due to chronic glomerulonephritis in 22 cases, chronic pyelonephritis in 10 cases, and unknown reasons in 2 cases.

In all patients, conventional treatment-including calcium supplementation, dietary restriction of phosphatebinding agents, and pulsed oral/intravenous vitamin D sterol therapy-failed to decrease the serum PTH level. Two criteria were established as the indications to perform the surgery. The first criterion was severe hyperparathyroidism (PTH level $>800 \mathrm{pg} / \mathrm{ml}$ in all cases) associated with hypercalcemia and/or hyperphosphatemia that was refractory to conventional treatment. The second criterion was the presence of clinical symptoms. In the ETP+AT group, we performed surgery in $21(61.8 \%)$ patients with pruritus, $28(82.4 \%)$ with bone and joint pain, $7(20.6 \%)$ with muscle weakness, $4(11.8 \%)$ with progressive soft tissue calcification, and $1(2.9 \%)$ with spontaneous fractures.

Serum calcium, phosphate, and total alkaline phosphatase were determined by standard methods. The serum PTH level (normal $15-65 \mathrm{pg} / \mathrm{ml}$ ) was $>800 \mathrm{pg} / \mathrm{ml}$ in all cases, with the highest being $>2500 \mathrm{pg} / \mathrm{ml}$. Serum calcium levels (normal $2.15-2.65 \mathrm{mmol} / \mathrm{l}$ ) ranged from 2.27 to 3.32 $\mathrm{mmol} / \mathrm{l} ; 27$ patients $(79.4 \%)$ had hypercalcemia (serum calcium $>2.63 \mathrm{mmol} / \mathrm{l}$ ), and the remaining patients were normal or had slight hypercalcemia. Hypercalcemia was considered to be a consequence of treatment with vitamin D or vitamin D metabolites. Serum phosphorous acid (normal $0.86-1.86 \mathrm{mmol} / \mathrm{l}$ ) ranged between 1.3 and $3.4 \mathrm{mmol} / \mathrm{l}$. Six patients had normal phosphorous acid levels, and the remaining patients had increased levels. Normal level of Serum alkaline phosphatase levels were normal $(<150 \mathrm{U} / \mathrm{l})$ in four patients, and the remaining patients had elevated levels.

Preoperative localization was based primarily on parathyroid ultrasonography (US) and ${ }^{99 \mathrm{~m}} \mathrm{Tc}$-sestamibi radionuclide scans, both of which were performed for all patients before the operation. The sensitivities of US and ${ }^{99 \mathrm{~m}} \mathrm{Tc}$-sestamibi radionuclide scans in determining the location and number of parathyroid glands were 73.5 and $85.3 \%$, respectively. Computed tomography (CT) was not a routine examination but was used when necessary. Plain radiographic examination of hands, feet, chest, and pelvis were performed to identify the lesions of renal osteodystrophy. Bone biopsy was not performed.

All patients were on chronic hemodialysis and were hemodialyzed the day before surgery and 1 or 2 days after the surgery. Prior to parathyroid surgery, all patients were administered with calcitriol to decrease secretion of PTH.

The procedure of ETP+AT is follows. Under general anesthesia, the patient was placed in the lithotomy position with the neck elevated by a sandbag. The surgery operator stood between the legs of the patient and an assistant stood at the right side of the patient. Three incisions were made at the crossing point of the bilateral medioclavicular lines, parasternal line, and the second intercostal level with the length of each incisions being $0.5,0.5$, and $1 \mathrm{~cm}$, respectively (Fig. 1). We created a subcutaneous space by injecting $1 \%$ epinephrine solution, an expanding dilator, and carbon dioxide $\left(\mathrm{CO}_{2}\right)$ with a pressure of $8 \mathrm{mmHg}$; we then placed three trocars according to the size of the incisions. Hypodermic porous connectivum of the thorax and cervical area was dissociated by an ultrasonic scalpel; the edge was on the lateral border of both sternocleidomastoid muscles and the inferior margin of thyroid cartilage. A longitudinal incision of the linea alba cervicalis was made, and the bilateral infrahyoid muscle groups were transected to expose the thyroid gland. Th posterior surface of the thyroid was explored to find the parathyroid glands (Fig. 2) according to the surgical anatomy of the parathyroids and the preoperative image diagnosis. The hyperplastic parathyroids were dissected, removed (Fig. 3), and preserved in ice. A portion of each parathyroid was examined and confirmed by pathological analysis. The cervical 


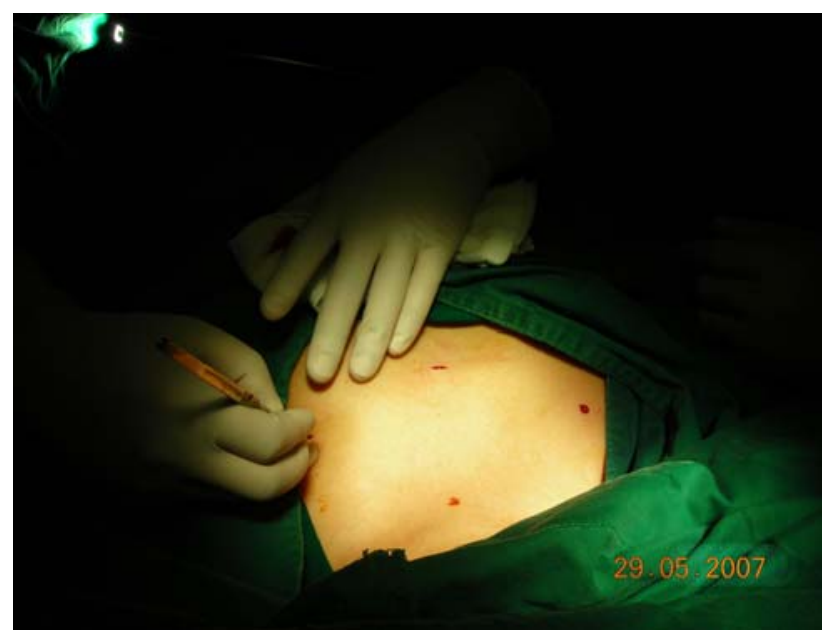

Fig. 1 Choice of incisions. Three incisions were made at the crossing point of the bilateral medioclavicular lines, parasternal line, and second intercostal level, with the length of the incisions being 0.5 , 0.5 , and $1.0 \mathrm{~cm}$, respectively

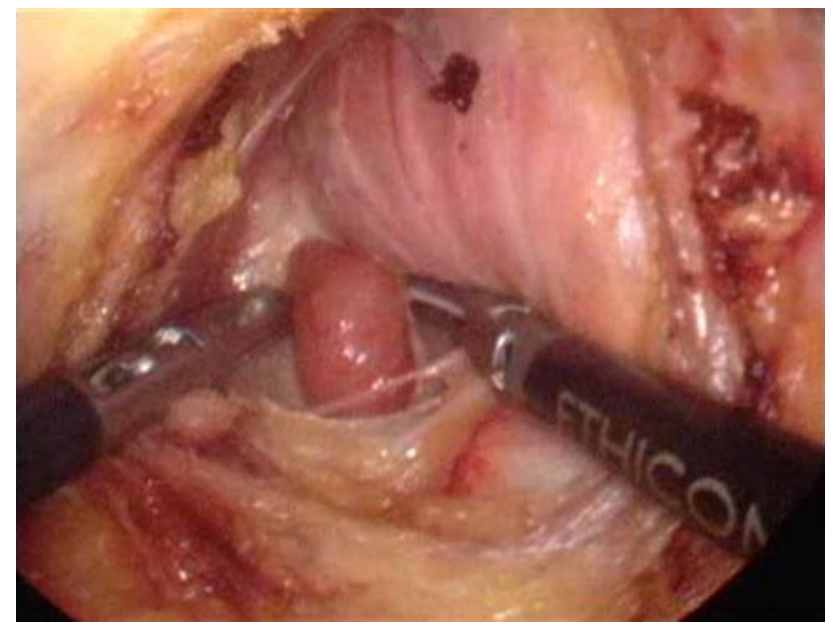

Fig. 2 Exposure of the parathyroids. The posterior surface of the thyroid was explored to search for the parathyroid glands

muscle that had been cut was sutured with 3-0 absorbable intermittent stitches after two drainage tubes were placed. For autotransplantation, 30 pieces of the sliced hyperplastic glands $\left(1 \times 1 \times 1 \mathrm{~mm}^{3}\right)$ were implanted into muscles in the forearm without formation of an arteriovenous $(\mathrm{A}-\mathrm{V})$ fistula for hemodialysis (Fig. 4a, b).

Hemodialysis was performed on the first or second day after surgery. Serum calcium levels were tested at 8 -h intervals over the first $48 \mathrm{~h}$ after the operation and then tested every day until they had stabilized. Supplemental calcium was given intravenously or orally. Vitamin D was provided on the first day after the operation and continued for 6 to 8 months.

Postoperative recurrence was diagnosed if symptoms such as pruritus, bone and joint pain, or others occurred and

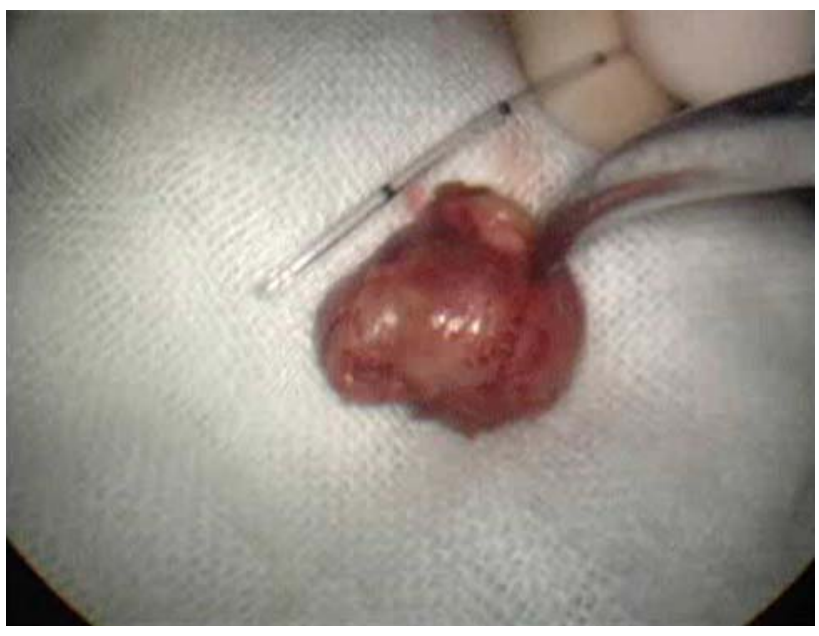

Fig. 3 Excised parathyroid glands. Hyperplastic parathyroids were dissected and removed
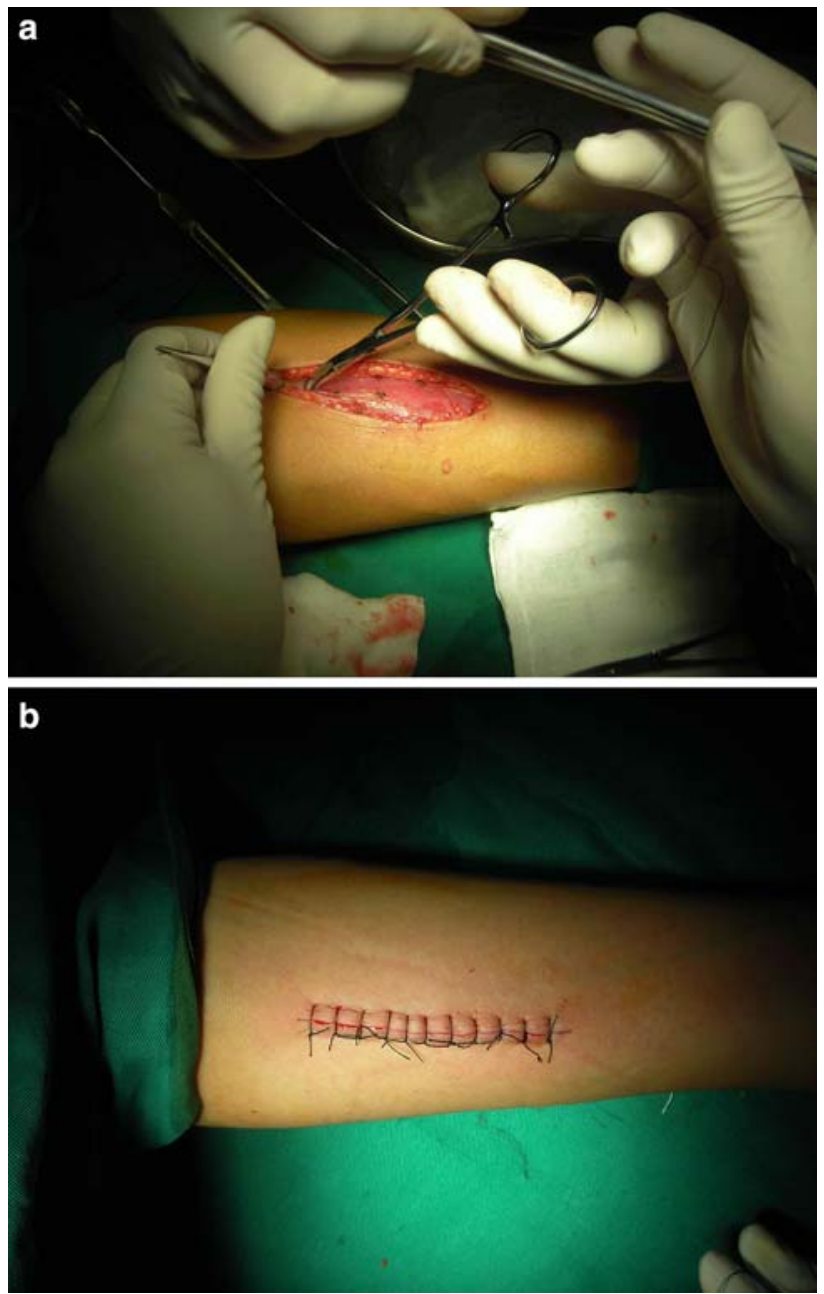

Fig. 4 a, b Autotransplantation. Sliced hyperplastic parathyroid tissues were implanted in forearm muscles 
the serum PTH level increased to $>200 \mathrm{pg} / \mathrm{ml}$. US and ${ }^{99 \mathrm{~m}} \mathrm{Tc}$ sestamibi scans helped us identify high uptake behind the thyroid and/or in the forearm.

\section{Results}

The average follow-up period after operation was 1.8 years (1 month to 3 years). The number of excised parathyroid glands was six in 2 patients, four in 29 patients, three in 2 patients and two in 1 patient. The weight of the removed glands ranged from 230 to $1280 \mathrm{mg}$ (median $790 \mathrm{mg}$ ). The average time of the operation was $2.5 \mathrm{~h}$, and the average volume of bleeding was approximately $20 \mathrm{ml}$. The clinical data are compared between ETP+AT and $\mathrm{TP}+\mathrm{AT}$ in Table 1.

There was no surgery-associated mortality, and the incidence of postoperative complications was relatively low. Two patients with high serum alkaline phosphatase levels before operation were found to have asymptomatic postoperative hypocalcemia; however, the hypocalcemia responded to intravenous injection of calcium gluconate and high doses of calcitriol and dialysate calcium. There was no postoperative bleeding that required surgical intervention and no permanent hypocalcemia. Recurrence of hyperparathyroidism occurred in one patient who had a fifth parathyroid gland hidden behind the thyroid, and resection was performed. Unfortunately, we found a sixth parathyroid behind the thyroid after the second operation and are currently preparing for a third resection.

Among the 34 EPT + PT operations, only one was converted to the traditional open operation, which was because of intraoperative bleeding. Using the traditional open operation in this case, bleeding was prevented and four parathyroids were successfully removed. No laryngeal nerves were apparently injured during the operation, although vocal hoarseness was observed afterward. These symptoms were relieved after conservative treatment. This

Table 1 Comparison of clinical data between ETP + AT and TP + AT

\begin{tabular}{lllc}
\hline Parameter & ETP+AT & TP+AT & $P$ \\
\hline Sex (male/female) & $15 / 19$ & $13 / 20$ & $>0.05$ \\
No. of excised parathyroid glands & 136 & 128 & $>0.05$ \\
Duration of the operation (h) & $2.5 \pm 0.9$ & $2.9 \pm 1.4$ & $>0.05$ \\
Intraoperative blood loss (ml) & 20 & 80 & $<0.05$ \\
Postoperative bleeding & 0 & 0 & - \\
Conversion to open surgery & $1(2.9 \%)$ & - & - \\
Hoarseness & $1(2.9 \%)$ & $1(3.0 \%)$ & $>0.05$ \\
Recurrence & $1(2.9 \%)$ & $3(9.1 \%)$ & $<0.05$ \\
Hospital stay (days) & $4.3 \pm 1.1$ & $6.5 \pm 1.8$ & $<0.05$
\end{tabular}

ETP endoscopic total parathyroidectomy, $A T$ autotransplantation patient experienced temporary hoarseness and recovered within 2 months. No septic complications were noted in the neck or forearm wounds.

The clinical symptoms and biochemical index of SHPT patients after ETP+AT were significantly improved. Pruritus was relieved in 18 of 21 patients $(85.7 \%)$, bone pain disappeared or was significantly relieved in 26 of 28 patients $(92.9 \%)$, and myopathy disappeared in all patients within 4 months after the operation. Calcium depositions in soft tissues either disappeared or were significantly diminished within 6 months after parathyroidectomy. There was no occurrence of pathologic bone fractures. By the third postoperative day, the serum calcium level dropped to within or below the normal range in all patients except the one with the recurrences. During the follow-up years, the average (mean $\pm \mathrm{SD}$ ) levels were as follows: serum calcium $2.24 \pm 0.12 \mathrm{mmol} / \mathrm{l}$, serum phosphorus acid $1.16 \pm 0.35 \mathrm{mmol} / \mathrm{l}$, and serum alkaline phosphatase $145 \pm 68 \mathrm{U} / \mathrm{l}$. In 33 of all of the patients, the serum PTH decreased to within the normal range.

Supplementation of vitamin D and calcium for the patients could be terminated or reduced after the grafted tissues took over the function of the glands. Recurrence of graft-dependent hyperparathyroidism was not observed in any of the patients.

Radiologic examination showed that osteodystrophy and soft tissue calcifications were relieved for most $(66.7 \%)$ of the patients 1 or 2 years after the surgery.

\section{Discussion}

Despite of the improvements in medical management, severe SHPT remains a common complication of CRF. According to a report of the European Dialysis and Transplant Association, parathyroidectomy is required in $15 \%$ of patients after 10 years of dialysis and in $38 \%$ of patients after 20 years of dialysis [10].

The primary indication for surgery in SHPT patients is an elevated serum PTH level. All 34 patients in the EPT +AT arm of our study had severe hyperparathyroidism (PTH $>800 \mathrm{pg} / \mathrm{ml}$ ). Conventional medical therapy had failed to decrease the PTH level, or it was complicated by the occurrence of hypercalcemia, which requires discontinuation of calcium/vitamin D therapy. Similar results were reported by Salem [11] in a survey of 612 chronic hemodialysis patients; he reported that $50 \%$ of patients had serum PTH levels that were three times higher than normal despite conventional medical therapy to prevent hyperparathyroidism. Hypercalcemia also enhances the need for surgery in SHPT patients $[5,7,8]$. In our study, 27 patients had hypercalcemia, and 28 had hyperphosphatemia. The clinical symptoms of SHPT that were refractory to 
conventional medical treatment include pruritus, bone and joint pain, muscle weakness, progression of soft tissue calcification, and spontaneous bone fractures.

Many authors have recommended that imaging of parathyroid glands is required only prior to reoperation. They also proposed that parathyroid imaging is not usually needed before the initial parathyroidectomy [12]. Others, however, have considered a preoperative imaging diagnosis highly useful for detecting parathyroid glands, especially those that are ectopically located [13, 14]. The most important point of the imaging diagnosis is to visualize all the parathyroid glands, including supernumerary glands that are located mainly in fat surrounding the glands or within the thymus or around the carotid artery $[15,16]$. Generally, supernumerary glands are found in $15 \%$ to $20 \%$ of patients $[16,17]$. We routinely performed US and ${ }^{99 \mathrm{~m}} \mathrm{Tc}$ scintigraphy in all patients before the operation to identify all of the supernumerary and/or ectopic parathyroid glands. ${ }^{99 \mathrm{~m}} \mathrm{Tc}$-sestamibi showed higher sensitivity for functionaccentuated localization of diseased glands. By using the sestamibi scan, we successfully identified all of the hyperplastic tissues in 29 patients, 2 hyperplastic tissues in 1 patient, and 3 hyperplastic tissues in 3 patients; only one patient had false-negative result for hyperplastic tissue. US was our primary localization procedure as it is noninvasive and not cost-intensive; and it was successful in localizing the parathyroids in 25 patients. We also found that US was more helpful for identifying parathyroid glands during the operation. Finally, CT scan was not an essential examination for the surgery, whereas plain radiographic examination of the hands, feet, chest, and pelvis was needed to identify lesions due to renal osteodystrophy.

There are many variations in parathyroidectomy procedures, especially with endoscopic surgery. To prevent recurrence, it is essential to remove all of the hyperplastic parathyroids, and a sufficient quantity of tissue must be used for transplantation.

Endoscopic bilateral exploration was performed in the patients in our study to identify all the parathyroid glands. Except one case that was converted to open operation, we removed 132 parathyroid glands from 33 patients. Among the 33 cases, we removed only three parathyroid glands in two patients and only two parathyroid glands in one patient. Follow-up observation showed that the treatment was satisfactory, and there was not hyperthyreosis. This suggests that not everyone has four parathyroid glands [18]. The operation (ETP) became more difficult without the sense of finger touch. Luckily, good endoscopic illumination made up this deficiency in some degree and avoided bleeding, which otherwise might cloud the surgical field and make it difficult for the operator to finish the surgery. In our study, one operation was converted to conventional surgery because of bleeding and injury of the recurrent laryngeal nerve after the operation. Therefore, less bleeding is of crucial importance to ensure the success of ETP. Blunt dissection is a major procedure in finding the parathyroid because the ultrasonic scalpel can lead to conductive injury of nerves and/or vessels. Postoperative bleeding that required surgical intervention was not observed in our study. Mild asymptomatic hypocalcemia was observed in two cases, even though they were given oral calcium supplements and calcitriol. None of our patients suffered from respiratory diseases due to edema or injury of the recurrent laryngeal nerve, although temporary hoarseness was observed in one patient. Finally, no septic complications were noted in the neck or forearm wounds. There was no recurrence of hyperplastic parathyroid tissue in the forearm muscles, possibly due to the short follow-up periods. Even if it occurs, though, we can easily excise it under local anesthesia or inject anhydrous alcohol to destroy the hyperplastic parathyroid tissues after localization with US and ${ }^{99 \mathrm{~m}} \mathrm{Tc}$ sestamibi scan.

In our study, bone pain was mostly found periarticularly in the shoulders, ankles, and heels, although sometimes it was generalized. Quick relief of bone pain was noted in $92.9 \%$ of patients within days after the operation. Pruritus disappeared after surgery in $85.7 \%$ of the patients. Myopathy disappeared in all patients, and soft tissue calcium deposits either disappeared or were markedly diminished within months after the surgery (Table 2).

Table 2 Comparison of biochemical detection for patients before and after ETP+AT

\begin{tabular}{lccccc}
\hline Biochemical measurement & Normal range & Before operation & After operation & \\
\cline { 3 - 5 } & & & 1 Day & 1 Week & 3 Months \\
\hline PTH $(\mathrm{pg} / \mathrm{ml})$ & $15-65$ & $1775.59 \pm 88.94$ & $112.62 \pm 337.77$ & $67.84 \pm 50.64$ & $51.49 \pm 24.75$ \\
BUN $(\mathrm{mmol} / \mathrm{l})$ & $2.10-7.20$ & $24.32 \pm 8.20$ & $21.85 \pm 10.34$ & $19.32 \pm 7.45$ & $22.83 \pm 5.34$ \\
$\mathrm{Cr}(\mu \mathrm{mol} / \mathrm{l})$ & $36.0-144.0$ & $862.53 \pm 242.07$ & $934.49 \pm 174.40$ & $903.54 \pm 19.47$ & $813.71 \pm 252.38$ \\
$\mathrm{Ca}(\mathrm{mmol} / \mathrm{l})$ & $2.15-2.65$ & $2.61 \pm 0.36$ & $2.01 \pm 0.19$ & $2.18 \pm 0.52$ & $2.24 \pm 0.12$ \\
P $(\mathrm{mmol} / \mathrm{l})$ & $0.86-1.86$ & $2.55 \pm 0.58$ & $1.34 \pm 0.32$ & $1.29 \pm 0.54$ & $1.16 \pm 0.35$ \\
ALP $(\mathrm{U} / \mathrm{l})$ & $15-150$ & $899.28 \pm 358.87$ & $422.32 \pm 129.04$ & $210.64 \pm 44.95$ & $145.05 \pm 68.27$ \\
\hline
\end{tabular}

$P T H$ parathyroid hormone, $B U N$ blood urea nitrogen, $C r$ creatinine; $P$ phosphorous acid, $A L P$ alkaline phosphatase 


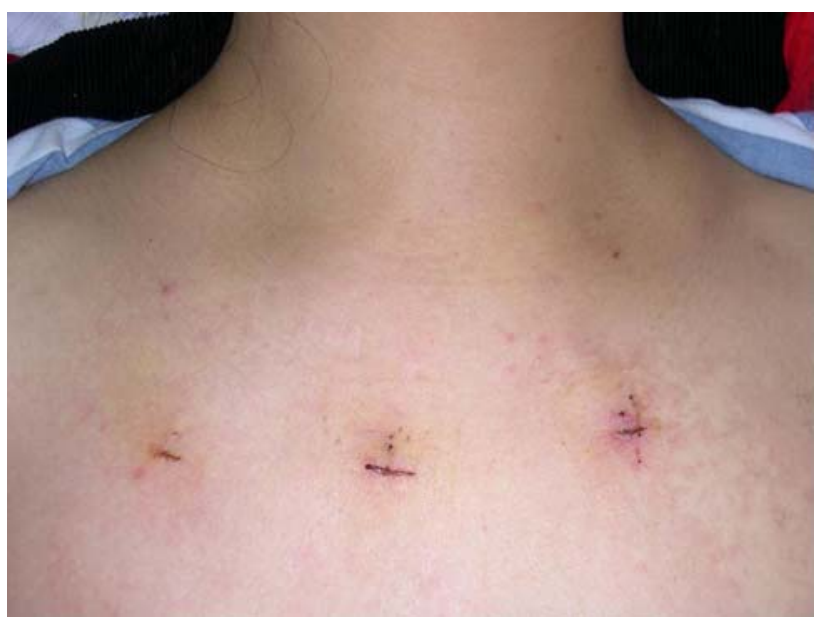

Fig. 5 Postoperative incisions

\section{Conclusions}

Endoscopic total parathyroidectomy with autotransplantation in the forearm is a highly effective, adequate treatment for advanced renal hyperparathyroidism. ETP + AT is a safe operation with low morbidity and mortality, shorter hospital stay, excellent outlook (Fig. 5), and low recurrence rate. It is important to identify and remove all the parathyroid glands and choose proper and adequate parathyroid tissue for autografting to prevent recurrence of hyperparathyroidism.

Open Access This article is distributed under the terms of the Creative Commons Attribution Noncommercial License which permits any noncommercial use, distribution, and reproduction in any medium, provided the original author(s) and source are credited.

\section{References}

1. Cohen EP, Moulder JE (2001) Parathyroidectomy in chronic renal failure: has medical care reduced the need for surgery? Nephron 89:271-274

2. Albright F, Baird PC, Cope O et al (1934) Studies on the physiology of parathyroid glands. IV. Renal complications of hyperparathyroidism. Am J Med Sci 187:49-65
3. Ganesh SK, Stack AG, Levin NW et al (2001) Association of elevated serum PO (4), $\mathrm{Ca} \times \mathrm{PO}$ (4) product, and parathyroid hormone with cardiac mortality risk in chronic hemodialysis patients. J Am Soc Nephrol 12:2131-2138

4. Raggi P, Boulay A, Chasan-Taber S et al (2002) Cardiac calcification in adult hemodialysis patients: a link between end-stage renal disease and cardiovascular disease? J Am Coll Cardiol 39:695-701

5. Llach F (1995) Secondary hyperparathyroidism in renal failure: the trade-off hypothesis revisited. Am J Kidney Dis 25:663-679

6. Akizawa T, Fukagawa M, Koshikawa S et al (1993) Recent progress in management of secondary hyperparathyroidism of chronic renal failure. Curr Nephrol Hypertens 2:558-565

7. Fournier A, Moriniere PH, Oprisiou R et al (1995) 1-alpha-Hydrovyvitamin D3 derivatives in the treatment of renal bone diseases: justification and optimal modalities of administration. Nephrology 71:254-283

8. Llach F (1990) Parathyroidectomy in chronic renal failure: indications, surgical approach and use of calcitriol. Kidney Int Suppl 29:62-68

9. Gourgiotis S, Moustafellos P, Stratopoulos C et al (2006) Total parathyroidectomy with autotransplantation in patients with renal hyperparathyroidism: indications and surgical approach. Hormones (Athens) 5:270-275

10. Fassbinder W, Brunner FP, Brynger H et al (1991) Combined report on regular dialysis and transplantation in Europe. Nephrol Dial Transplant 6:5-35

11. Salem MM (1997) Hyperparathyroidism in the hemodialysis population a survey of 612 patients. Am J Kidney Dis 29:862865

12. Chang CW, Tsue TT, Hermreck AS et al (2000) Efficacy of preoperative dual phase sestamibi scanning in hyperparathyroidism. Am J Otolaryngol 21:355-359

13. Takagi H, Tominaga $Y$, Uchida $K$ et al (1983) Image diagnosis of parathyroid glands in chronic renal failure. Ann Surg 198:74-79

14. Takagi H, Tominaga Y, Uchida K et al (1986) Evaluation of image diagnosing methods of enlarged parathyroid glands in chronic renal failure. World J Surg 10:605-611

15. Numano M, Tominaga Y, Uchida K et al (1998) Surgical significance of supernumerary parathyroid glands in renal hyperparathyroidism. World J Surg 22:1098-1103

16. Akerstr $\varphi m$ G, Malmaeus J, Bergstr $\varphi$ m R (1984) Surgical anatomy of human parathyroid glands. Surgery 95:14-21

17. Wang CA (1976) The anatomic basis of parathyroid surgery. Ann Surg 183:271-274

18. Hibi Y, Tominaga Y, Uchida K et al (2002) Cases with fewer than four parathyroid glands in patients with renal hyperparathyroidism at initial parathyroidectomy. World J Surg 26:314317 